\title{
Dinâmica da invasão de Pinus elliottii Engelm. em restinga sob processo de restauração ambiental no Parque Florestal do Rio Vermelho, Florianópolis, SC
}

\author{
Kurt Bourscheid* \\ Ademir Reis \\ Laboratório de Restauração Ambiental Sistêmica \\ Departamento de Botânica, Universidade Federal de Santa Catarina \\ Campus Trindade, CEP 88040-900, Florianópolis - SC, Brasil \\ *Autor para correspondência \\ bourscheid@gmail.com
}

Submetido em 10/08/2009

Aceito para publicação em 02/03/2010

\section{Resumo}

A invasão de espécies em comunidades naturais representa uma grande ameaça à biodiversidade global. A segunda maior causa de extinção de espécies no mundo está relacionada com a ação de espécies invasoras, superada apenas pela perda e fragmentação de habitats. $\mathrm{O}$ objetivo deste estudo foi quantificar os níveis de re-infestação de Pinus elliottii Engelm var. elliottii no Parque Florestal do Rio Vermelho, Florianópolis, SC. Um ano após a derrubada de um hectare de Pinus, foram montadas 100 parcelas para avaliar a regeneração de Pinus. Avaliações semestrais foram feitas do inverno de 2003 ao verão de 2005 . A densidade populacional apresentou diferenças estatísticas, variando de 19.617 indivíduos/ha até 166.624 indivíduos/ha. O efeito de borda evidenciou-se a partir do inverno de 2004 e aumentou significativamente a taxa de emergência. As faixas mais próximas da borda apresentaram maiores densidades populacionais, chegando a 362.268 indivíduos/ ha até $10 \mathrm{~m}$ da borda. A média da densidade populacional para cada faixa apresentou uma escala exponencial decrescente que vai de 569.598 indivíduos/ha na faixa mais próxima à borda a 43.768 indivíduos/ha na faixa mais distante da borda. Sugere-se cortar os indivíduos em estágio Jovem 2 a cada 4 anos, aproximadamente, de forma a controlar a reinfestação.

Unitermos: dinâmica populacional, invasão biológica, restauração ambiental

\section{Abstract}

Invasion dynamics of Pinus elliottii Engelm. on restinga under a restoration process inside Parque Florestal do Rio Vermelho, Florianópolis, SC. Species invasion in natural communities is considered a great threat to global biodiversity. The second biggest cause of extinction worldwide is related to invasive species action, which is only smaller than habitat loss and fragmentation. The objective of this study was to quantify the levels of Pinus elliottii Engelm. var. elliottii infestation in Parque Florestal do Rio Vermelho, Florianópolis, SC, Brazil. One year after the clearance of one hectare of Pinus, 100 plots were set to evaluate Pinus regeneration. Semestral evaluations were made between the winter of 2003 and the summer of 2005 . Population density showed significant differences $(\mathrm{p}<0.05)$, varying from 19,617 plants/ha to $166,624 \mathrm{plants} / \mathrm{ha}$. The border effect became strong after the winter of 2004 and the emergence rate increased significantly. The zones closest to the 
border showed the highest population densities, reaching 362,268 plants/ha on the first 10m from the border. The mean population density of each zone resulted in a decreasing exponential equation where there were 569,598 plants/ha on the zone closest to the border and 43,768 plants/ha on the border's furthest zone. This led to the recommendation to cut all Young 2 plants about every 4 years in order to control the reinfestation.

Key words: biological invasion, population dynamics, restoration

\section{Introdução}

A contaminação biológica é conceituada como o processo de introdução e adaptação de espécies exóticas que se naturalizam (através da adaptação gradativa dos mecanismos biológicos das exóticas), tornando-se invasoras e provocando mudanças nos ecossistemas naturais (Ziller, 2000). A segunda maior causa de extinção de espécies no mundo está relacionada com a ação de espécies invasoras, superada apenas pela perda e fragmentação de habitats (Brasil, 2002; IBGE, 2004; Liesenfeld e Pellegrim, 2004), sendo o gênero Pinus o maior causador de extinções por invasão (Bechara, 2003).

Grande número de espécies de plantas foram introduzidas e cultivadas em novas regiões com fins ornamentais, agrícolas ou para pastagem, ignorando o filtro histórico (Lambers et al., 1998) que impedia que tais espécies ocorressem nestas regiões. Muitas dessas espécies deixaram de ser cultivadas e se estabeleceram na comunidade local, superando, assim, o filtro fisiológico (Lambers et al., 1998) que só permite a sobrevivência das epécies adaptadas ao ambiente local. Segundo Primack e Rodrigues (2001), algumas espécies são frequentemente transportadas acidentalmente, assim como as sementes de ervas daninhas que acompanham acidentalmente outras sementes colhidas ou mudas para fins comerciais e que depois acabam sendo semeadas em outras localidades, como é o caso do arroz vermelho. Estes autores também sugerem que o capim-gordura (Mellinis minutiflora) teria chegado ao Brasil aderido à roupa dos escravos.

Lake e Leishman (2003) citam algumas características que provavelmente sejam responsáveis pela ação invasora das plantas, tais como área da superfície foliar, textura e pilosidade das folhas, massa das sementes, forma de crescimento, modo de dispersão, capacidade de propagação vegetativa, época de floração e altura do dossel. Rejmànek e Richardson (1996) compararam a capacidade de invasão de várias espécies de Pinus e concluíram que sementes de pequena massa, período juvenil curto e pequenos intervalos entre os ciclos reprodutivos são características de táxons com alta capacidade invasora. Entretanto, de acordo com Petit et al. (2004), parece não haver uma "síndrome invasora".

Porém, Keane e Crawley (2002) argumentam que um mecanismo comumente aceito de invasão é dado pela "hipótese da exclusão de inimigos", também chamada de exclusão da herbivoria, fuga de predadores ou exclusão ecológica. Assim, uma espécie introduzida em novo ambiente faz menos interações com a comunidade local que as espécies nativas.

Em ilhas, as plantas introduzidas de folhas lignificadas e não palatáveis, podem sobreviver melhor à presença de herbívoros do que as plantas nativas mais tenras e, desse modo, as espécies exóticas chegam a dominar a paisagem à medida que a vegetação nativa diminui (Primack e Rodrigues, 2001).

Hurlbert (1971) salienta que a importância ecológica de cada uma das espécies dentro das comunidades está associada com a sua capacidade de promover "probabilidades de interações interespecíficas". A contaminação biológica ocorre exatamente porque as espécies invasoras apresentam um mínimo de interações, uma vez que não evoluíram se relacionando com os organismos de seus novos ambientes. Além disso, Brown (1987) coloca que numa comunidade em que houve um processo natural de colonização, não existem espécies inimigas umas das outras, pois os consumidores são os principais responsáveis pelo controle populacional das espécies. Assim, de acordo com o mesmo autor, estas espécies são contidas dentro de seus padrões demográficos, de forma a manter uma maior diversidade possível dentro dos ambientes naturais.

$\mathrm{O}$ gênero Pinus tem sido muito estudado no sul do Brasil como planta produtora de madeira (Seitz e Corvello, 1983; Jankovski, 1996; Mattei e Seitz, 1996), 
mas só em anos mais recentes estas plantas têm sido estudadas como invasoras (Ziller, 2000; Bechara, 2003; Ziller e Galvão, 2003; Liesenfeld e Pellegrim, 2004; Cattaneo, 2005).

De alguma forma, a população de Pinus parece exercer um efeito de inibição (Ricklefs, 1996) sobre as espécies que participam dos estágios iniciais da sucessão após uma perturbação. Este efeito diminui a resiliência do ambiente e dificulta o processo de restauração.

Segundo Bechara (2003), o gênero Pinus permite poucas probabilidades de encontros interespecíficos, o que evita seu controle demográfico através da função dos consumidores, quebrando o equilíbrio entre produtores e consumidores e formando uma comunidade dominada por poucas espécies.

Além disso, áreas ocupadas por Pinus durante muito tempo possuem uma espessa camada de acículas que se acumula na serapilheira. De acordo com Sturgess (1991), as acículas de Pinus são de difícil degradação, pois, apesar de possuírem grande quantidade de celulose, possuem baixa concentração de nitrogênio. Também apresentam ceras cuticulares que reduzem os efeitos da ação mecânica e possuem compostos polifenólicos que mantêm as proteínas em formas resistentes à ação microbiana. Essa dificuldade na degradação causa a formação de uma camada de até $20 \mathrm{~cm}$ de serapilheira que, de acordo com o mesmo autor, causa podsolização do solo devido à alta acidez das acículas. Além disso, Kim e Shin (2005) demonstraram o efeito antibacteriano de compostos voláteis de Pinus densiflora Siebold \& Zucc.

Vieira (2004) ressalta que o banco de sementes de Pinus do Parque Florestal do Rio Vermelho apresenta viabilidade curta no solo (cerca de três meses), porém, forma um banco de plântulas que contribui, pela sua quantidade, para uma efetiva invasão da restinga local.

Devido aos impactos gerados pelo Pinus elliottii Engelm. var. elliottii no Parque Florestal do Rio Vermelho, Bechara (2003) montou uma Unidade Demonstrativa de $10.000 \mathrm{~m}^{2}$ (1ha) onde, em Junho de 2002, foi realizado o corte raso de todos os indivíduos de Pinus sp. para desenvolver técnicas para a substituição destes pela vegetação de restinga original da área.

Segundo Bechara (2003), a chuva de sementes de Pinus elliottii Engelm. var. elliottii é constante na área de estudo, chegando a mais de 3 milhões de sementes dispersadas por hectare por ano, com $90 \%$ de emergência de plântulas. Desta maneira, qualquer ação de restauração na região será submetida a um intenso processo de reinfestação por Pinus elliottii var. elliottii.

O contato entre a Unidade Demonstativa e o talhão adjacente pode ser definido como uma borda (Scariot et al., 2003), local onde podem ocorrer importantes alterações na temperatura do solo e do ar, na umidade relativa, na umidade do solo, na intensidade de luz e na velocidade do vento (Yates et al., 2004), conhecidas como efeito de borda. Tomimatsu e Ohara (2004) e Yates et al. (2004) ressaltam que tais alterações microclimáticas podem modificar o recrutamento de diversas espécies.

Diante dos impactos ambientais oriundos do plantio de espécies invasoras do gênero Pinus, torna-se prioritário estudar a dinâmica ecológica do mesmo, assim como o comportamento ecológico da vegetação natural frente ao caráter agressivo deste gênero. $\mathrm{O}$ conhecimento da biologia e ecologia das plantas invasoras é essencial para se efetuar o manejo das áreas contaminadas, visando o controle e, enfim, a erradicação das populações de plantas invasoras.

O objetivo deste estudo foi quantificar os níveis de re-infestação e avaliar a dinâmica do crescimento de Pinus elliotii Engelm. var. elliottii em uma Unidade Demonstrativa de restauração ambiental da vegetação de restinga, no Parque Florestal do Rio Vermelho, Florianópolis, SC.

\section{Material e Métodos}

A Ilha de Santa Catarina está situada entre as latitudes $27^{\circ} 22^{\prime} 45^{\prime \prime}$ e $27^{\circ} 50^{\prime} 10^{\prime \prime} \mathrm{S}$ e as longitudes $48^{\circ} 21^{\prime} 37^{\prime \prime}$ e 48 $34^{\prime} 49^{\prime \prime} W$ no litoral do Estado de Santa Catarina. A Unidade Demonstrativa (Bechara, 2003) utilizada como área de estudo encontra-se dentro do Parque Florestal do Rio Vermelho (PFRV), situado no nordeste da Ilha de Santa Catarina. Originalmente ocupado por restinga arbórea, foi utilizado, em 1962, para o plantio experimental de 25 espécies de Pinus e três espécies de Eucalyptus (Caruso, 1983). Localiza-se entre o Oceano Atlântico e a Lagoa da Conceição, perfazendo 
uma área de 1.465ha. A leste do PFRV encontra-se a Praia de Moçambique (12,5km de extensão) e a oeste, a Lagoa da Conceição.

O clima da Ilha de Santa Catarina é do tipo subtropical úmido, com temperatura média anual de $20^{\circ} \mathrm{C}$. A precipitação total anual é de $1.400 \mathrm{~mm}$, e a umidade relativa anual é de 80-85\% (Santa Catarina, 1986).

No Parque Florestal do Rio Vermelho há quase 500ha de talhões de Pinus spp. e encontra-se hoje com aproximadamente 250 ha de dunas e restingas contaminadas. Os impactos ambientais oriundos do processo de contaminação biológica por Pinus na restinga do PFRV são evidentes e conspícuos. Além disso, a alta capacidade invasora e regenerativa do gênero é nitidamente visível em outras unidades de conservação contaminadas por Pinus do município de Florianópolis, SC: o Parque Municipal das Dunas da Lagoa da Conceição, a Unidade de Conservação Ambiental do Desterro e a Estação Ecológica de Carijós (Bechara, 2003).

Dentro de uma Unidade Demonstrativa (1ha) foram plotados 10 transectos de $10 \mathrm{~m}$ espaçados em $10 \mathrm{~m}$, e nestes foram implantadas parcelas permanentes circulares de raio igual a $1 \mathrm{~m}$, perfazendo um total de 100 parcelas distribuídas sistematicamente. Essas parcelas foram montadas demarcando-se o centro de cada uma com estacas de bambu.

As plântulas de Pinus presentes em cada uma das parcelas foram contadas, etiquetadas e mapeadas. Foram realizadas quatro avaliações semestrais de Julho 2003 a Janeiro de 2005. As avaliações foram realizadas de acordo com o seguinte cronograma:

- Avaliação 01: Julho/Agosto de 2003

- Avaliação 02: Janeiro/Fevereiro de 2004

- Avaliação 03: Julho/Agosto de 2004

- Avaliação 04: Janeiro/Fevereiro de 2005

Em cada uma das avaliações os indivíduos novos foram demarcados e incluídos nas avaliações.

As etiquetas utilizadas foram pequenas plaquetas de alumínio contendo o número da parcela, o número do indivíduo dentro da parcela e o símbolo da estação quando a planta foi registrada no campo.
Foram estabelecidos estádios de crescimento para os indivíduos avaliados adaptando-se a proposta de Gatsuk et al. (1980), onde foram considerados:

a) Plântula - indivíduos apenas com os cotilédones.

b) Jovem 1 - indivíduos com os cotilédones e início do desenvolvimento de folhas.

c) Jovem 2 - indivíduos sem cotilédones e somente com folhas.

Para analisar as consequências do efeito de borda sobre a densidade populacional a área foi dividida em cinco faixas concêntricas de 10 a $50 \mathrm{~m}$ da borda.

A densidade populacional da área e das faixas foi submetida à análise de variância e, quando o resultado do F-teste foi significativo $(p<0,05)$, foi aplicado o teste SNK para separação de médias.

A taxa de mortalidade foi calculada observando-se qual a porcentagem de indivíduos que morreram de uma avaliação para outra. As consequências do efeito de borda foram analisadas em função da densidade populacional em relação à distância da borda do talhão.

Os coeficientes de variação das amostras foram calculados para cada período de avaliação e para cada faixa a partir da borda, obtidos através da fórmula $\mathrm{CV}=100 \mathrm{~s} / \mathrm{m}$ onde, $\mathrm{s}=$ desvio padrão da amostra e $\mathrm{m}$ $=$ média da amostra.

Foi utilizada uma média do número de indivíduos médio de cada avaliação em cada faixa para a elaboração da equação de regressão. A densidade nas diferentes faixas foram enquadradas em uma função exponencial por ter sido o modelo significativo com o melhor ajuste $\left(\mathrm{R}^{2}\right)$, cuja fórmula é $\mathrm{y}=\exp (\mathrm{a}+\mathrm{bx})$ onde, $\mathrm{y}=$ densidade populacional e $\mathrm{x}=$ distância da borda.

As análises de variância (ANOVA) e as equações de regressão foram realizadas com a utilização do software Statgraphics 7.0 e SAS (Statistical Analisys System) 8.01.

\section{Resultados}

A densidade populacional na primeira avaliação resultou em 19.617 indivíduos/ha (CV=143,11\%). Na segunda avaliação ocorre uma diminuição da densidade 
que não chega a ser significativa $(\mathrm{p}<0,05)$, sendo de 14.490 indivíduos/ha (CV=133,61\%). A terceira avaliação foi a única significativamente $(\mathrm{p}<0,05)$ diferente e apresentou 166.624 indivíduos/ha (CV=188,66\%) (Tabela 1). $\mathrm{Na}$ quarta avaliação a densidade caiu para 68.535 indivíduos/ha $(\mathrm{CV}=186,27 \%)$, indicando baixa produção se sementes e uma alta mortalidade neste período.

As taxas de mortalidade foram maiores no verão, chegando 58,73\% (Tabela 1) na quarta avaliação. A segunda avaliação também apresentou elevada taxa de mortalidade com $35,23 \%$.

$\mathrm{Na}$ coorte da primeira avaliação, apenas $23,70 \%$ eram Plântulas e mais da metade (51,79\%) era Jovem 2. Na avaliação 02 houve a passagem de $11,37 \%$ das Plântulas para os estádios seguintes e 58,76\% de Jovem
2. Da terceira avaliação em diante, o banco de plântulas passou a ser exclusivamente de indivíduos Jovem 2 (Tabela 2).

TABELA 1: Avaliação da densidade populacional e taxa de mortalidade de Pinus elliottii var. elliottii no Parque Florestal do Rio Vermelho, Florianópolis, SC. As letras (a, b) representam diferenças significativas no teste de separação de médias SNK.

\begin{tabular}{ccc} 
Período & $\begin{array}{c}\text { Densidade pop. } \\
\text { (ind./ha) }\end{array}$ & $\begin{array}{c}\text { Taxa de mortalidade } \\
\text { (\%) }\end{array}$ \\
\hline Jul./Ago. 2003 & $19.617 \mathrm{a}$ & 0,00 \\
Jan./Fev. 2004 & $14.490 \mathrm{a}$ & 35,23 \\
Jul./Ago. 2004 & $166.624 \mathrm{~b}$ & 9,89 \\
Jan./Fev. 2005 & $68.535 \mathrm{a}$ & 58,73 \\
\hline
\end{tabular}

TABELA 2: Porcentagem de indivíduos em cada estádio ontogenético em cada avaliação e porcentagem de indivíduos de Pinus vivos e mortos em cada momento.

\begin{tabular}{|c|c|c|c|c|c|c|c|c|c|}
\hline & & Avaliação 01 & & Avaliação 02 & & Avaliação 03 & & Avaliação 04 & \\
\hline & Estágio & Vivas & Mortas & Vivas & Mortas & Vivas & Mortas & Vivas & Mortas \\
\hline Jul/Ago 2003 & Plântulas & $23,70 \%$ & - & - & $12,33 \%$ & - & - & - & - \\
\hline $\mathrm{N}=616$ & Jovem 1 & $24,51 \%$ & - & $6,05 \%$ & $6,47 \%$ & - & $0,72 \%$ & - & - \\
\hline \multirow[t]{17}{*}{$100 \%$} & Jovem 2 & $51,79 \%$ & - & $58,76 \%$ & $16,39 \%$ & $59,01 \%$ & $5,08 \%$ & $50,46 \%$ & $8,55 \%$ \\
\hline & Total & $100 \%$ & - & $64,81 \%$ & $35,19 \%$ & $59,01 \%$ & $40,99 \%$ & $50,46 \%$ & $49,54 \%$ \\
\hline & & & Estágios & Vivas & Mortas & Vivas & Mortas & Vivas & Mortas \\
\hline & & Jan/Fev 2004 & Plântulas & - & - & - & - & - & - \\
\hline & & $\mathrm{N}=57$ & Jovem 1 & $8,77 \%$ & - & - & $1,75 \%$ & - & - \\
\hline & & $100 \%$ & Jovem 2 & $91,23 \%$ & - & $85,97 \%$ & $12,28 \%$ & $70,17 \%$ & $15,78 \%$ \\
\hline & & & Total & $100 \%$ & - & $85,97 \%$ & $14,03 \%$ & $70,17 \%$ & $29,83 \%$ \\
\hline & & & & & Estágios & Vivas & Mortas & Vivas & Mortas \\
\hline & & & & Jul/Ago 2004 & Plântulas & $56,53 \%$ & - & - & $43,65 \%$ \\
\hline & & & & $\mathrm{N}=4852$ & Jovem 1 & $42,15 \%$ & - & - & $21,48 \%$ \\
\hline & & & & $100 \%$ & Jovem 2 & $1,32 \%$ & - & $34,46 \%$ & $0,41 \%$ \\
\hline & & & & & Total & $100 \%$ & - & $34,46 \%$ & $65,54 \%$ \\
\hline & & & & & & & Estágios & Vivas & Mortas \\
\hline & & & & & & Jan/Fev 2005 & Plântulas & $97,94 \%$ & - \\
\hline & & & & & & $N=146$ & Jovem 1 & - & - \\
\hline & & & & & & $100 \%$ & Jovem 2 & $2,06 \%$ & - \\
\hline & & & & & & & Total & $100 \%$ & - \\
\hline
\end{tabular}


$\mathrm{Na}$ coorte da segunda avaliação não houve plântulas, mas 91,23\% de Jovem 2, sendo que logo na avaliação seguinte só restaram indivíduos Jovem 2 . Na coorte da terceira avaliação, 56,53\% eram Plântulas e $42,15 \%$ eram Jovem 1, o que sugere a ocorrência de uma alta produtividade de sementes neste período. Além disso, apenas 12,88\% das Plântulas passou para Jovem 2 na avaliação seguinte e não houve indivíduos Jovem 1. Na coorte da avaliação 04 , houve uma população de Plântulas de 97,94\% (Tabela 2) de um total de 146 indivíduos.

$\mathrm{Na}$ análise do efeito de borda, as faixas de $10 \mathrm{~m}$ e $20 \mathrm{~m}$ de distância da borda do talhão apresentaram densidade populacional significativamente $(\mathrm{p}<0,001)$ superior às demais faixas, sendo que na avaliação 03 havia 362.268 indivíduos/ha (CV=126,55\%) e 84.224 indivíduos/ ha $(\mathrm{CV}=95,29 \%)$ em cada faixa, respectivamente. Observando-se a média da densidade populacional para cada faixa pode-se notar uma escala exponencial nitidamente decrescente que vai de 569.598 indivíduos/ ha na faixa mais próxima à borda a 43.768 indivíduos/ha na faixa mais distante da borda (Figura 1).

\section{Discussão}

Observando-se as coortes de cada período, pode-se perceber uma nítida diferença na porcentagem de cada estádio de crescimento entre as coortes da primeira e terceira avaliações. O aumento da quantidade de cones nas matrizes mais próximas da área de estudo (observação pessoal) e o grande aumento na proporção de Plântulas na coorte da terceira avaliação sugere que a população analisada sofreu as consequências do efeito de borda. Acredita-se que a coorte observada na primeira avaliação seja proveniente de sementes que iniciaram sua formação ainda no inverno de 2002, apenas um ou dois meses após a criação da Unidade Demonstrativa, que ocorreu em Maio de 2002, e que vieram a emergir somente no inverno de 2003. Segundo Suassuna (1977), o tempo de maturação do estróbilo feminino é muito variável, dependendo da umidade e temperatura locais. Sendo assim, pode-se considerar que no PFRV o estróbilo feminino leva aproximadamente um ano para completar sua maturação, no entanto, testes mais específicos precisam ser realizados neste sentido. Desta forma, é possível que, no inverno de 2003, as matrizes

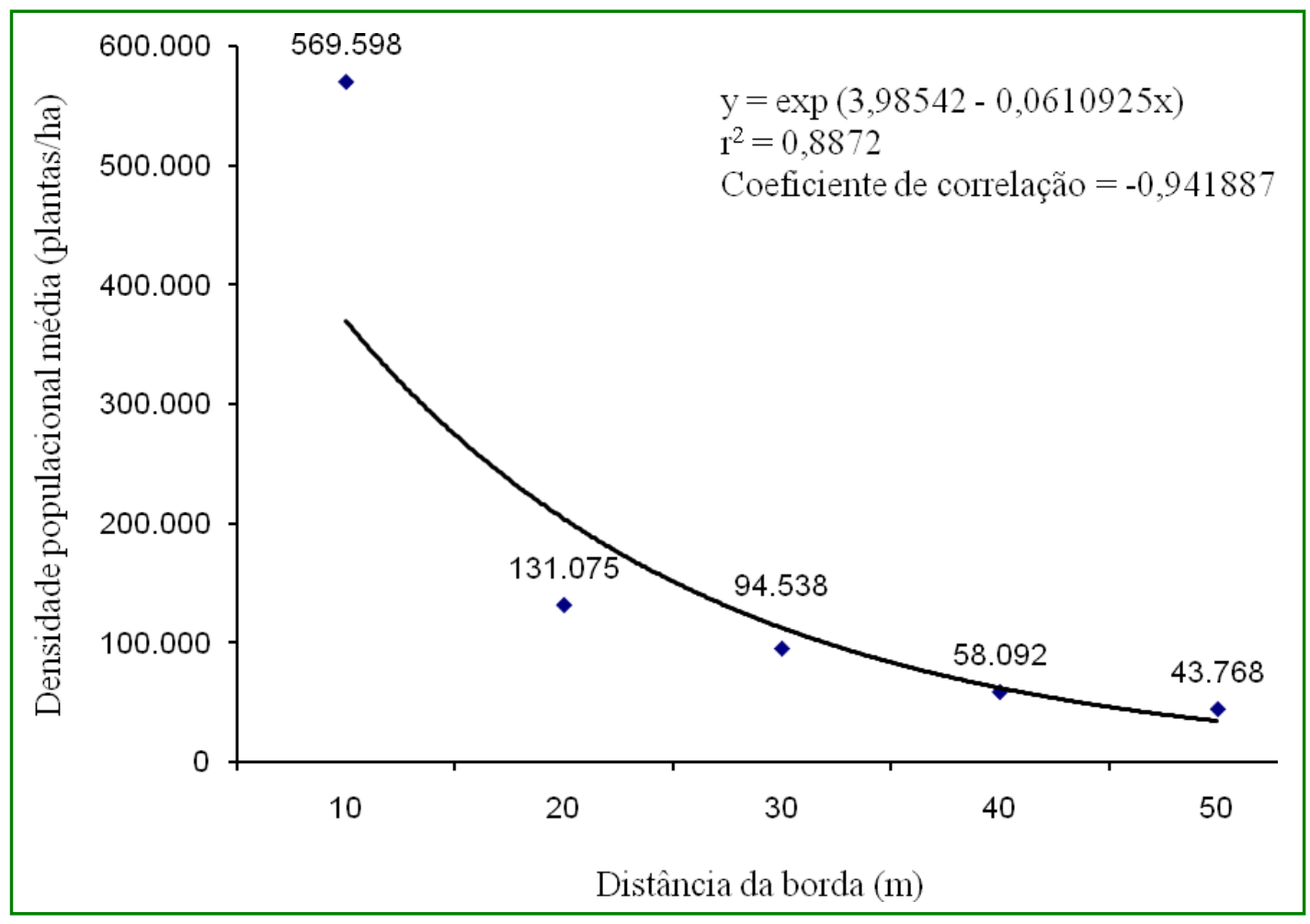

FIGURA 1: Densidade populacional média de plântulas de Pinus em função da distância da borda talhão. 
tenham respondido às alterações microclimáticas com o aumento na produção de sementes, que emergiram no inverno de 2004. Além disso, a baixa longevidade do banco de sementes e a alta porcentagem de germinação reforçam a hipótese de que um aumento na densidade populacional está relacionado a um aumento na produção de sementes.

O desenvolvimento das plântulas desde sua emergência ocorreu de forma bastante rápida, sendo que grande parte das plântulas observadas em estádio de Plântula em uma determinada avaliação encontravam-se mortas ou em estádio de Jovem 2 na avaliação seguinte. Esta alta velocidade de desenvolvimento pode ser um dos fatores responsáveis pela alta capacidade invasora do gênero Pinus. A ausência de Plântulas na segunda avaliação é um forte indício de que dentro de um talhão ocorre uma produção muito baixa de sementes no verão e da baixa longevidade do banco de sementes.

Os dados da primeira coorte sugerem que um ano após a emergência das plântulas, o banco de plântulas estabiliza-se no estádio de Jovem 2, e passa a apresentar menores taxas de mortalidade que no estádio de Plântula. Entretanto, parece haver uma alta taxa de mortalidade nos períodos de verão, em resposta às altas taxas de emergência encontradas no inverno.

Quanto às estimativas sobre as densidades populacionais em relação à distância da borda do talhão, já era esperada que a maior densidade fosse observada aos $10 \mathrm{~m}$ da borda, onde a chuva de sementes é mais intensa devido à proximidade das matrizes, seguido da faixa de $20 \mathrm{~m}$ e assim sucessivamente.

A ação antrópica permitiu que o gênero Pinus chegasse ao Parque Florestal do Rio Vermelho, fazendo-o passar pelo filtro histórico que naturalmente evitaria que propágulos deste gênero chegassem à região. As características deste gênero (principalmente Pinus elliottii var. elliottii) permitiram uma rápida adaptação às condições ambientais encontradas no Parque, permitindo que passasse pelo filtro fisiológico que eliminaria do ambiente espécies não adaptadas, e passando a se reproduzir. Em seguida o Pinus passou pelo filtro biológico (Lambers et al., 1998) e passou a interagir com a comunidade local, dominando-a.
Os impactos ambientais oriundos do plantio de espécies invasoras do gênero Pinus, tornam essencial fundamentar ações de controle desta invasora na restauração ecológica de ambientes contaminados, conscientização ambiental e políticas públicas para eliminar o processo de contaminação biológica. $\mathrm{O}$ planejamento da restauração necessita da informação dos níveis de re-infestação para que sejam tomadas decisões sobre quanto e quando as plantas deverão ser eliminadas de modo a não impedirem o processo de re-colonização das plantas nativas de restinga. Muitas técnicas de restauração de áreas degradadas baseadas em nucleação (Reis et al., 2003) vêm sendo aplicadas na área numa tentativa de minimizar os efeitos da re-infestação e restaurar a restinga arbórea original.

A análise dos estádios de crescimento é importante para propor um programa de corte do Pinus de forma mais barata e eficiente, evitando gastos excessivos e competição prejudicial ao processo regenerativo da restinga. Além disso, conhecer os níveis de sobrevivência das plântulas de Pinus spp. sobre o talhão explorado é fundamental para subsidiar o planejamento da retirada das plântulas re-infestantes após a exploração dos talhões.

Tendo em vista os dados obtidos neste trabalho, considera-se importante realizar intervenções na área a fim de promover o corte e eliminação dos indivíduos que se encontrarem no estádio Jovem 2. Sugere-se que este processo seja realizado a cada 4 anos aproximadamente, até que seja restaurada a resiliência do ambiente e a própria comunidade seja capaz de evitar a invasão.

Considerando-se o possível aumento da produção de sementes nas bordas do talhão, sugere-se que talhões utilizados para o cultivo de Pinus sejam cercados por vegetação nativa que possa reduzir o efeito de borda nestes talhões, de forma a minimizar a invasão de novos ambientes.

Espera-se que o presente trabalho sirva de base para trabalhos de recuperação e restauração ambiental de outras áreas contaminadas com espécies do gênero Pinus. Contudo, estudos complementares precisam ser desenvolvidos para planejar com maior precisão as intervenções a serem realizadas no processo de invasão. 


\section{Agradecimentos}

Ao $\mathrm{CNPq}$ pelo apoio financeiro, à equipe do Laboratório de Restauração Ambiental Sistêmica, Prof ${ }^{a}$. Tânia Tarabini Castellani, Alexandre Siminski e Ângelo Puchalski pelas discussões teóricas.

\section{Referências}

Bechara, F. C. 2003. Restauração ecológica de restingas contaminadas por Pinus no Parque Florestal do Rio Vermelho, Florianópolis, SC. Dissertação de Mestrado, Universidade Federal de Santa Catarina, Brasil, 125pp.

Brasil. 2002. Impactos sobre a biodiversidade. Disponível em <http://www.mma.gov.br/biodiversidade/biodiv/perda.html $>$. Acesso em 28 de novembro de 2002.

Brown, Jr. K. 1987. O papel dos consumidores na conservação e no manejo in situ. IPEF, 37: 61-69.

Caruso, M. M. L. 1983. O desmatamento da Ilha de Santa Catarina de 1500 aos dias atuais. $1^{\text {a }}$ ed. Editora da UFSC, Florianópolis, Brasil, 158pp.

Cattaneo, M. 2005. La disperción de coniferas exóticas en areas naturales: Ejemplos de Nueva Zelanda. Disponível em $<\mathrm{http} / /$ www.institutohorus.org.br/download/artigos/conifinvasorasNZ. pdf $>$. Acesso em 18 de maio de 2005.

Gatsuk, L. E.; Smirnova, O. V.; Vorontzova, L. I.; Zaugolnova, L. B.; Zhukova, L. A. 1980. Age states of plants of various growth forms: a review. Journal of Ecology, 68: 675-696.

Hurlbert, S. 1971. The nonconcept of species diversity: A critic and alternative parameters. Ecology, 52 (4): 577-586.

IBGE. 2004. Dimensão ambiental - biodiversidade. Indicadores de Desenvolvimento Sustentável - Brasil 2004. Disponível em $\quad<$ http://www.institutohorus.org.br/download/artigos/ibge biodiversidade.PDF>. Acesso em 19 de maio de 2005.

Jankovski, T. 1996. Estudo de alguns aspectos da regeneração natural induzida em povoamentos de Pinus taeda L. e Pinus elliottii Engelm. Floresta, 26 (1/2): 95-96.

Keane, R. M.; Crawley, M. J. 2002. Exotic plant invasions and the enemy release hipothesis. Trends in Ecology and Evolution, 17 (4): 164-170.

Kim, Y.; Shin, D. 2005. Volatile components and antibacterial effects of pine needle (Pinus densiflora S. and Z.) extracts. Food Microbiology, 22: 37-45.

Lake, J. C.; Leishman, M. R. 2003. Invasion success of exotic plants in natural ecosystems: the role of disturbance, plant attributes and freedom from herbivores. Biological Conservation, 117: 215-226.

Lambers, H.; Stuart, F.; Pons, T. 1998. Plant Physiological Ecology. $2^{\mathrm{a}}$ ed. Springer-Verlag, New York, USA, 540pp.

Liesenfeld, M. V. A.; Pellegrim, L. M. 2004. Risco ecológico: A invasão por Pinus e a problemática das espécies alienígenas vegetais no Parque Estadual de Itapuã - Viamão, RS. Disponível em <www.institutohorus.org.br/download/artigos/LIESENFELD_ PELOTAS_2004.pdf $>$. Acesso em 18 de maio de 2005.
Mattei, V. M.; Seitz, R. A. 1996. Crescimento inicial de plantas de Pinus taeda L. originadas por semeadura direta no campo. Floresta, 26 (1/2): 45-58.

Petit, R. J.; Bialozyt, R.; Garnier-Géré, P.; Hampe, A. 2004. Ecology and genetics of tree invasions: from recent introductions to Quaternary migrations. Forest and Ecology Management, 197: 117-137.

Primack, R. B.; Rodrigues, E. 2001. Biologia da Conservação. $1^{\text {a }}$ ed. Editora Midiograf. Londrina, Brasil, 328pp.

Reis, A.; Bechara, F. C.; Espíndola, M. B.; Vieira, N. K.; Souza, L. L. 2003. Restauração de áreas degradadas: a nucleação como base para incrementar os processos sucessionais. Natureza \& Conservação, 1 (1): 28-36.

Rejmànek, M.; Richardson, D. M. 1996. What attributes make some plant species more invasive? Ecology, 77: 1655-1661.

Ricklefs, R. E. 1996. A Economia da Natureza. $3^{a}$ ed. Editora Guanabara Koogan SA., Rio de Janeiro, Brasil, 470pp.

Santa Catarina. 1986. Atlas de Santa Catarina. $1^{\text {a }}$ ed. Governo do Estado de Santa Catarina. Gabinete de Planejamento e Coordenação Geral Rio de Janeiro, Florianópolis e Rio de janeiro, Brasil, 173pp.

Scariot, A.; Freitas, S. R.; Neto, E. M.; Nascimento, M. T.; Oliveira, L. C.; Sanaiotti, T.; Sevilha, A. C.; Villela, D. M. 2003. Vegetação e Flora. In: Rambaldi, D. M. \& Oliveira, D. A. (Orgs). Fragmentação de Ecossistemas: Causas, efeitos sobre a biodiversidade e recomendações de políticas públicas. Ministério do Meio Ambiente/Secretaria de Biodiversidade e Florestas, Brasília, Brasil, p.103-123.

Seitz, R. A.; Corvello, W. V. 1983. A regeneração natural de Pinus elliottii em área de campo. Anais do Simpósio sobre Florestas Plantadas nos Neotrópicos como Fonte de Energia, Universidade Federal de Viçosa, Viçosa, Brasil, p. 48-51.

Sturgess, P. W. 1991. Post-felling vegetation changes on three afforested sand-dune systems. $\mathrm{PhD}$ Thesis, University of Liverpool, UK, 244pp.

Suassuna, J. 1977. A cultura do Pinus: Uma perspectiva e uma preocupação. Disponível em <www.fundaj.gov.br/docs/tropico/ desat/pinus.html>. Acesso em 07 de junho de 2005.

Tomimatsu, H.; Ohara, M. 2004. Edge effects on recruitment of Trillium camschatcense in small forest fragments. Biological Conservation, 117: 509-519.

Vieira, N. K. 2004. O papel do banco de sementes na restauração de restinga sob talhão de Pinus elliottii Engelm. Dissertação de Mestrado, Universidade Federal de Santa Catarina, Brasil, 77pp.

Yates, E. D.; Levia Jr.; D. F.; Wiliams, C. L. 2004. Recruitment of three non-native plants into a fragmented forest in southern Illinois. Forest Ecology and Management, 190: 119-130.

Ziller, S. R. 2000. A Estepe gramíneo-lenhosa no segundo planalto do Paraná: diagnóstico ambiental com enfoque à contaminação biológica. Tese de Doutorado, Universidade Federal do Paraná, Brasil, 268pp.

Ziller, S. R.; Galvão, F. 2003. A degradação da estepe gramíneolenhosa no Paraná por contaminação biológica de Pinus elliotti e $P$. taeda. Revista Floresta, 32 (1): 41-47. 